\section{Perfil Lipidico de Pacientes Com Alto Risco para Eventos Cardiovasculares na Prática Clínica Diária}

\begin{abstract}
RESUMO
Embora existam recomendações especificas envolvendo o tratamento das dislipidemias em pacientes com alto risco, estas recomendações dificilmente são seguidas adequadamente. O objetivo deste estudo é investigar fatores de risco em pacientes com alto risco cardiovascular acompanhados ambulatorialmente no Brasil e Venezuela. Os prontuários de 412 pacientes foram selecionados em 4 instituições. Os pacientes foram divididos conforme a utilização de hipolipemiantes. Pacientes sem hipolipemiantes apresentavam níveis mais elevados de colesterol total $(p<0,001)$, LDL colesterol $(p<0,001)$ e HDL colesterol $(p<$ $0,001)$, além de menores níveis de triglicérides $(p<0,001)$. $O$ uso de hipolipemiantes foi associado à diminuição dos niveis de colesterol total $(251,0 \pm 40,0$ para 196,0 $\pm 46,0)$, LDL colesterol $(168,0 \pm 36,0$ para $116,0 \pm$ $39,0), \mathrm{HDL}$ colesterol $(51,0 \pm 46,0$ para $46,0 \pm 12,0)$ e triglicérides $(181,0 \pm$ $120,0$ para $160,0 \pm 79,0)$. Concluímos que apenas um pequeno percentual de pacientes, mesmo em uso de estatinas, apresenta níveis de colesterol compativel com os atualmente recomendados. Desta forma, embora as recomendações para tratamento das dislipidemias sejam bem conhecidas, um pequeno percentual de pacientes atinge os valores desejados de colesterol. É necessário um melhor controle dos níveis lipídicos dos pacientes, tanto através da utilização de doses maiores de estatinas como da utilização da associação de hipolipemiantes. (Arq Bras Endocrinol Metab 2006;50/3:481-489)
\end{abstract}

Descritores: Dislipidemia; Estatinas; Drogas hipolipemiantes; Diabetes mellitus; Doença cardiovascular

\begin{abstract}
Lipid Profile of Patients with Increased Risk for Cardiovascular Events in Daily Clinical Practice.

Although there are specific guidelines regarding the treatment of dyslipidemia in highly risk patients, these recommendations are usually inadequately followed. The aim of this study is to investigate risk factors in patients with increased cardiovascular risk currently treated in Brazil and Venezuela. Medical charts of 412 patients were selected in 4 institutions. Patients were divided into groups according to the use of lipidlowering drugs (LLD), particularly statins. Patients who did not use LLD showed higher levels of total cholesterol $(p<0.001)$, LDL cholesterol $(p<$ $0,001)$ and HDL cholesterol $(p<0.001)$, besides lower levels of triglycerides $(p<0.001)$. The use of statins was associated with a decrease in levels of total cholesterol (from $251.0 \pm 40.0$ to $196.0 \pm 46.0$ ), LDL cholesterol (from $168.0 \pm 36.0$ to $116.0 \pm 39.0$ ), HDL cholesterol (from $51.0 \pm$ 46.0 to $46.0 \pm 12.0$ ) and triglycerides (from $181.0 \pm 120.0$ to $160.0 \pm 79.0$ ). Finally, only a small percentage of patients, even those under treatment with LLD, showed cholesterol levels according to currently available guidelines. Therefore, although the guidelines for the treatment of dyslipidemia are widely known, only a small percentage of patients achieve adequate levels of cholesterol. It is necessary to decrease
\end{abstract}

artigo original

Rodrigo O. Moreiva

Raul D. Santos

Lilton Martinez

Fabiana C. Saldanba

Jara Lucia A.C. Pimenta

Josefina Feijoo

Natalie Jabnke

Otávio C. Mangile

Rosane Kupfer

Instituto Estadual de Diabetes e Endocrinologia (ROM, FCS, JLACP, RK), Rio de Janeiro, RJ; Unidade Clínica de Dislipidemias do Instituto do Coração (InCor) do Hospital das Clínicas da Faculdade de Medicina da

Universidade de São Paulo (RDS, LM, OCM), São Paulo, SP; Ambulatório de Dislipidemias do Hospital do Servido Público Estadual de São Paulo, SP (LM) e Unidad Cardiológica Latinoamericana (JF, NJ), Caracas, Venezuela.

Recebido em 30/05/05

Revisado em 17/11/05 e 31/01/06 Aceito em 10/02/06 
lipid levels of these patients by increasing the dose of the statins or using a second drug. (Arq Bras Endocrinol Metab 2006;50/3:481-489)

Keywords: Dyslipidemia; Statins; Lipid-lowering drugs; Diabetes mellitus; Cardiovascular disease

A $S$ DOENÇAS CARDIOVASCULARES são hoje a maior causa de mortalidade no mundo $(1,2)$. Diversos estudos já foram realizados demonstrando que existem vários fatores diretamente relacionados à elevada incidência de eventos cardiovasculares, principalmente o tabagismo, a hipertensão arterial, a dislipidemia e o diabetes mellitus (DM) (3). A abordagem adequada destes fatores de risco está associada a uma diminuição na incidência e na progressão das doenças cardiovasculares.

Dentre os diversos fatores de risco para doença cardiovascular, a dislipidemia vem surgindo com um dos mais importantes. Diversos estudos randomizados e controlados com placebo já demonstraram que a diminuição dos níveis do colesterol total e LDL colesterol está associada a uma menor incidência de eventos cardiovasculares (Infarto Agudo do Miocárdio e Acidente Vascular Cerebral) (ver ref. 4 para revisão). Em particular, a utilização dos inibidores da HMG-CoA reductase (estatinas) vem se tornando mandatória em pacientes com elevado risco para aterosclerose, incluindo pacientes com DM tipo 2.

Os efeitos das estatinas nos níveis de colesterol são bem conhecidos. Seu uso está relacionado a uma diminuição nos níveis de colesterol total, LDL colesterol e triglicérides, além de um pequeno aumento nos níveis de HDL colesterol (5). Embora sua utilização seja amplamente recomendada hoje mesmo em pacientes com níveis considerados normais de colesterol (6), suas ações na prática clínica diária em ambientes não controlados ainda não foram adequadamente estudadas. Alguns autores sugerem que, embora os chamados clinical trials sejam considerados o padrão-ouro para a avaliação da eficácia e segurança das medicações, outras informações podem surgir apenas com a avaliação da prática clínica regular a longo prazo $(7,8)$.

O objetivo deste estudo é investigar diversos fatores de risco cardiovascular em uma população acompanhada ambulatorialmente em alguns centros de tratamento na América do Sul. Além disso, será também avaliado o impacto do uso dos hipolipemiantes nos níveis de colesterol conforme demonstrado por dados obtidos em prontuários.

\section{MATERIAL E MÉTODOS}

\section{População do estudo}

Foram analisados 412 prontuários em quatro diferentes centros de atendimento: Instituto Estadual de Diabetes e Endocrinologia do Rio de Janeiro (IEDE) $(\mathrm{n}=138)$, Instituto do Coração (InCor) do Hospital das Clínicas da Faculdade de Medicina da Universidade de São Paulo $(n=124)$, Hospital do Servidor Público Estadual de São Paulo (HSPE-SP) $(\mathrm{n}=84)$ e Unidad Cardiológica LatinoAmericana (UCLA) ( $\mathrm{n}=$ 66), Caracas, Venezuela. Neste estudo retrospectivo, o prontuário avaliado só era incluído na casuística se o caso do paciente pudesse ser classificado em pelo menos um dos grupos abaixo (critérios de inclusão) e se não preenchesse nenhum dos critérios de exclusão. O protocolo foi aprovado pelo Comitê de Ética em Pesquisa das Instituições. Por se tratar de um estudo de prontuários, foi dispensada a obtenção do Termo de Consentimento Livre e Esclarecido.

\section{Critérios de inclusão}

Os pacientes eram selecionados de modo a preencher pelo menos um dos 3 critérios de avaliação: Grupo I Pacientes diabéticos tipo 2 , sem história prévia de evento cardiovascular e/ou coronariopatia; Grupo II Pacientes com doença arterial coronariana, sem o diagnóstico de Diabetes Mellitus; Grupo III - Pacientes com DM tipo 2 e doença coronária.

O diagnóstico de doença arterial coronariana (DAC) era realizado caso o paciente preenchesse pelo menos 1 dos seguintes critérios: (i) Infarto Agudo do Miocárdio (IAM) prévio, confirmado através de pelo menos dois dos seguintes achados: dor precordial e/ou alteração no eletrocardiograma e/ou elevação de enzimas cardíacas; (ii) Acidente Vascular Cerebral (AVC) Isquêmico prévio, confirmado através de tomografia computadorizada e/ou ressonância magnética; (iii) Teste Ergométrico positivo e/ou cintilografia de perfusão miocárdica positivo e/ou angiografia coronária mostrando obstruções de pelo menos 50\% da luz do vaso; (iv) Angioplastia coronariana transluminal percutânea e/ou colocação de stent prévios e (vi) enxertos de veia safena ou artérias mamárias. O diagnóstico do DM era realizado conforme critérios da American Diabetes Association (9) em pacientes com mais de 45 anos.

\section{Critérios de exclusão}

Foi excluído da pesquisa o paciente que apresentasse, no prontuário, qualquer dos seguintes itens: Acidente Vascular Cerebral Hemorrágico Prévio; Doença 
endócrina predispondo à obesidade (tais como hipotireoidismo descompensado e Síndrome de Cushing); Doença renal (creatinina sérica $>1,8 \mathrm{mg} / \mathrm{dL}[159$ $\mathrm{mmol} / \mathrm{L}$ ]) ou hepática (enzimas hepáticas [AST, ALT], bilirrubinas ou fosfatase alcalina $>2,5$ vezes os valores de referência) clinicamente manifesta; história prévia de crise convulsiva e/ou uso de anticonvulsivantes; abuso de álcool ou drogas; antecedentes de cirurgia gastrintestinal com a finalidade de perda de peso; outras doenças sistêmicas significativas; pacientes em tratamento para Síndrome da Imunodeficiência Adquirida (uso de inibidores de protease e/ou inibidores da transcriptase reversa).

\section{Desenho do estudo}

Após avaliação dos critérios de inclusão e exclusão, o prontuário era pesquisado de modo a identificar o último perfil lipídico realizado pelo paciente. Os pacientes foram então divididos em dois grupos: (i) Sem uso de medicamentos hipolipemiantes: os pacientes que não utilizam ou utilizaram medicamentos hipolipemiantes tiveram avaliados apenas o perfil lipídico e os fatores de risco cardiovascular, conforme descrito posteriormente; (ii) Uso de medicamentos hipolipemiantes: os pacientes que utilizavam medicamentos hipolipemiantes tiveram seus prontuários revistos para a identificação dos exames e fatores de risco imediatamente anteriores ao início da medicação. Além disso, todas as alterações na terapia hipolipemiante (início de novo medicamento ou aumento de dose) durante o período de tratamento também foram avaliadas. $\mathrm{O}$ paciente era considerado em uso de hipolipemiantes se estivesse em uso de uma das seguintes classes de medicação: fibratos, resinas seqüestrantes de ácidos biliares, ácido nicotínico e estatinas. O desenho esquemático do estudo está apresentado na figura 1 .

\section{Variáveis analisadas}

As seguintes informações foram extraídas dos prontuários: idade, sexo, Índice de Massa Corporal (IMC) (calculado conforme a fórmula: peso/altura ${ }^{2}$ ), pressão arterial sistólica e diastólica, glicemia de jejum, hemoglobina glicosilada e perfil lipídico. É importante enfatizar que, embora tenham sido determinados nos laboratórios das diferentes instituições participantes, os parâmetros bioquímicos foram determinados por métodos similares.

\section{Análise estatística}

A análise estatística foi realizada com o programa GraphPad InStat 3.00 para Windows 95 (GraphPad Software, San Diego, California, USA). As comparações entre os diferentes grupos (DM tipo 2 vs. DAC vs. DM tipo $2+$ DAC) foram realizadas através de Análise de Variância (ANOVA) com post-tests (Teste de Bonferroni) para variáveis contínuas e o Teste do Qui-quadrado para variáveis categoriais. A comparação entre os pacientes em uso de hipolipemiantes com os que não utilizavam foi realizada com o Teste $t$ de Student não pareado. Em casos de amostras não paramétricas, foi aplicada a correção de Welch. Foram utilizados testes bicaudados em todas as comparações. As comparações entre os diversos fatores de risco antes do início da terapia hipolipemiante com a últimas consultas no prontuário foram realizadas com o teste $t$ de Student pareado. O limite de significância para todas as comparações foi de $5 \%$.

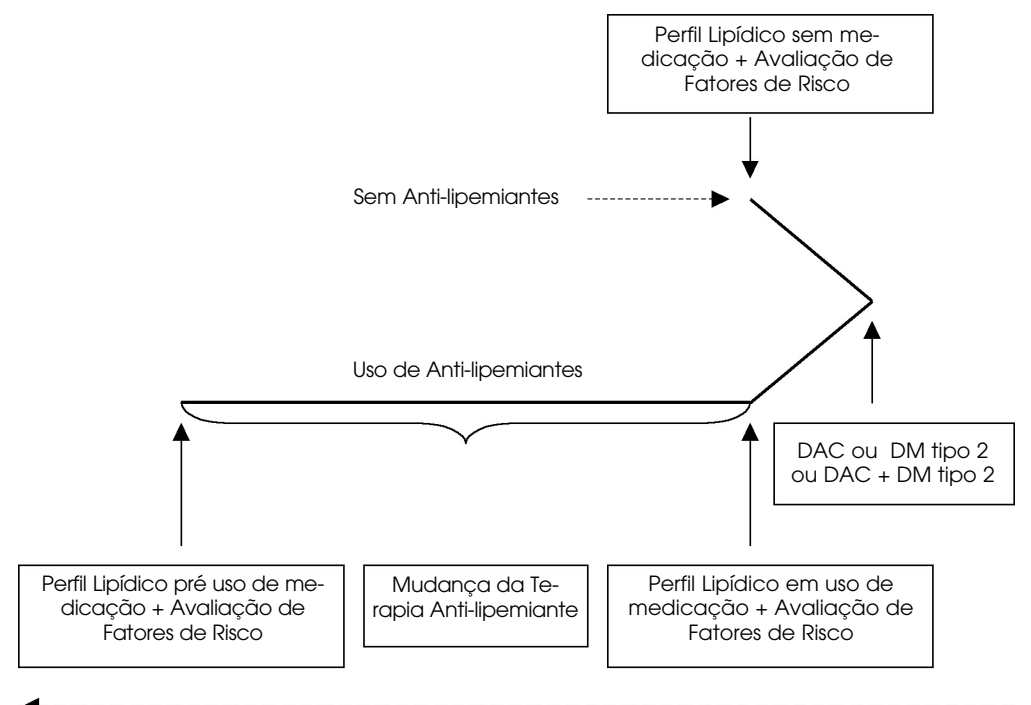

Figura 1. Desenho do estudo. 


\section{RESULTADOS}

Inicialmente, foram avaliadas as características dos pacientes de cada instituição. A prevalência de pacientes nos grupos 1, 2 e 3 foi de, respectivamente: no IEDE, $81,9 \%, 8,7 \%$ e $9,4 \%$; no InCor, $62,0 \%, 25,0 \%$ e $13,0 \%$; no HSPE-SP, 73,8\%, 16,6\% e 9,5\%; na UCLA, 57,6\%, $37,9 \%$ e $4,5 \%$. Embora tenha sido encontrada uma diferença na proporção dos grupos conforme a instituição, nenhuma diferença foi encontrada na comparação dos grupos (por exemplo, Grupo 1 do IEDE x Grupo 1 do InCor x Grupo 1 do HSPE-SP etc.), isto é, os grupo são homogêneos, independente da instituição avaliada (dados não mostrados).

A tabela 1 apresenta os diversos fatores de risco cardiovascular estudados conforme a distribuição dos pacientes em 3 grupos: Grupo 1 - pacientes com DM e ausência de DAC; Grupo 2 - paciente com DAC e ausência de DM e Grupo 3 - pacientes com DM e DAC. Pacientes do grupo 1 , apesar de serem mais novos, apresentam níveis mais elevados de pressão arterial diastólica (PAd), colesterol total, LDL colesterol e de HDL colesterol, associado a uma menor utilização de estatinas. A presença de doença cardiovascular, associada ou não ao $\mathrm{DM}$, pareceu estar relacionada a um melhor controle dos fatores de risco cardiovascular (PAd, colesterol total e LDL colesterol), com uma maior utilização de estatinas. Entretanto, pacientes com DAC apresentam também níveis menores de HDL colesterol (tabela 1).

Devido à grande importância do excesso de peso no DM e na DAC, a prevalência de sobrepeso $\left(\right.$ IMC $\geq 25$ e $<30 \mathrm{~kg} / \mathrm{m}^{2}$ ) e obesidade (IMC $\geq 30$ $\mathrm{kg} / \mathrm{m}^{2}$ ) foi comparada entre os grupos. A prevalência de sobrepeso foi de $39,1 \%$ no grupo $1,50 \%$ no grupo 2 e $39,5 \%$ no grupo 3 . Em relação à obesidade, a prevalência foi de $32,7 \%$ no grupo $1,28,0 \%$ no grupo 2 e $44,7 \%$ no grupo 3 . Estas diferenças, entretanto, não foram estatisticamente significantes $(\mathrm{p}>0,05$ para todas as comparações).

O impacto das alterações no metabolismo da glicose nos pacientes com DAC e sem DM também foi avaliado em nosso estudo. Desta forma, os pacientes do grupo 2 foram divididos em pacientes com glicemia normal $(<100 \mathrm{mg} / \mathrm{dl})$ ou com intolerância de jejum (glicemia $\geq 100$ e $<126 \mathrm{mg} / \mathrm{dl}$ ). Cinco pacientes não apresentavam dosagem de glicemia e foram excluídos, assim como 2 pacientes com glicemia acima de 126 $\mathrm{mg} / \mathrm{dl}$ (diagnóstico de DM). É importante observar que o uso de estatinas foi mais freqüente nos pacientes com intolerância de jejum, levando conseqüentemente a níveis menores de LDL colesterol (tabela 2).

A figura 2 apresenta o percentual de pacientes em cada grupo que atinge os níveis desejados no perfil lipídico, conforme recomendação das III Diretrizes Brasileiras de Dislipidemias e Prevenção da Aterosclerose (Triglicérides $<150 \mathrm{mg} / \mathrm{dl}, \mathrm{LDL}<100 \mathrm{mg} / \mathrm{dl} \mathrm{e}$ HDL $\geq 45 \mathrm{mg} / \mathrm{dl}$ ) (10) e do National Cholesterol Education Program Adult Treatment Panel III Guidelines (11). Nessa amostra populacional, a presença concomitante de DM e DCV relacionou-se a um maior percentual de pacientes que atingem os níveis recomendados de LDL colesterol. Entretanto, a porcentagem de pacientes com HDL $\geq 45 \mathrm{mg} / \mathrm{dL}$ foi significativamente menor nos pacientes com DAC, independente da presença de DM.

Os pacientes que fazem de uso de medicação hipolipemiante foram comparados com pacientes sem

Tabela 1. Parâmetros clínicos e bioquímicos da população estudada, conforme dados da última consulta disponível no prontuário.

\begin{tabular}{|c|c|c|c|}
\hline & $\begin{array}{l}\text { Diabetes Mellitus } \\
\text { (sem DCV) } \\
(n=288)\end{array}$ & $\begin{array}{l}\text { Diabetes Mellitus } \\
(\text { com DCV) } \\
(n=82)\end{array}$ & $\begin{array}{c}\text { Doença Cardiovascular } \\
(\text { sem DM) } \\
(n=40)\end{array}$ \\
\hline Idade (anos) & $60,4 \pm 9,6^{*}, U$ & $65,0 \pm 9,5$ & $65,5 \pm 8,4$ \\
\hline $\mathrm{IMC}\left(\mathrm{kg} / \mathrm{m}^{2}\right)$ & $28,2 \pm 4,9$ & $27,9 \pm 3,7$ & $30,0 \pm 5,5$ \\
\hline PAs (mmHg) & $143,0 \pm 24,0$ & $139,5 \pm 19,0$ & $140,0 \pm 19,0$ \\
\hline PAd $(\mathrm{mmHg})$ & $86,0 \pm 15,0^{*}$ & $85,0 \pm 9,0$ & $80,0 \pm 12,0$ \\
\hline Glicose (mg/dl) & $161,0 \pm 74,0$ & $104,0 \pm 23,0 \#, U$ & $142,0 \pm 55,0$ \\
\hline $\mathrm{HbAlc}(\%)$ & $8,1 \pm 1,9$ & $6,0 \pm 0,3 \#, U$ & $7,8 \pm 1,7$ \\
\hline Usa hipolipemiante (\%) & 22,5 & $82,9 \#, \cup$ & $60,0 \#$ \\
\hline Colesterol Total (mg/dl) & $214,0 \pm 45,0^{*}, U$ & $194,0 \pm 49,0$ & $182,0 \pm 44,0$ \\
\hline HDL Colesterol (mg/dl) & $53,0 \pm 14,0^{*}, U$ & $46,0 \pm 13,0$ & $43,0 \pm 10,0$ \\
\hline LDL Colesterol (mg/dl) & $131,0 \pm 41,0 U$ & $119,0 \pm 41,0$ & $108,0 \pm 39,0$ \\
\hline Triglicérides (mg/dl) & $151,0 \pm 91,0$ & $158,0 \pm 72,0$ & $135,0 \pm 61,0$ \\
\hline
\end{tabular}

DM: Diabetes Mellitus; DCV: Doença Cardiovascular; IMC: Índice de Massa Corporal; PAs: Pressão Arterial Sistólica; PAd: Pressão Arterial Diastólica

\# $\mathrm{p}<0,01 \times \mathrm{DM}$ (sem DCV); U $\mathrm{p}<0,05 \times \mathrm{DM}$ (com DCV); ${ }^{\mathrm{p}}<0,01 \times$ Doença Cardiovascular (sem DM) 
Tabela 2. Parâmetros clínicos e bioquímicos dos pacientes com doença arterial coronariana e sem diabetes mellitus conforme a presença de intolerância de jejum.

\begin{tabular}{|c|c|c|}
\hline & $\begin{array}{c}\text { Glicemia Normal } \\
(<100 \mathrm{mg} / \mathrm{dl}) \\
(n=39)\end{array}$ & $\begin{array}{c}\text { Intolerância Glicídica } \\
(\geq 100 \mathrm{e}<126 \mathrm{mg} / \mathrm{dl}) \\
(\mathrm{n}=34)\end{array}$ \\
\hline Idade (anos) & $65,0 \pm 10,3$ & $65,0 \pm 8,4$ \\
\hline $\mathrm{IMC}\left(\mathrm{kg} / \mathrm{m}^{2}\right)$ & $27,8 \pm 4,0$ & $27,9 \pm 3,6$ \\
\hline PAs (mmHg) & $136,5 \pm 20,0$ & $140,2 \pm 17,9$ \\
\hline PAd (mmHg) & $83,3 \pm 9,3$ & $85,1 \pm 8,1$ \\
\hline Usa hipolipemiante (\%) & 76,7 & $97,0^{*}$ \\
\hline Colesterol Total (mg/dl) & $190,3 \pm 48,9$ & $188,3 \pm 45,6$ \\
\hline HDL Colesterol (mg/dl) & $43,1 \pm 9,9$ & $49,4 \pm 15,2$ \\
\hline LDL Colesterol (mg/dl) & $124,6 \pm 37,1$ & $104,5 \pm 38,6^{*}$ \\
\hline Triglicérides (mg/dl) & $160,4 \pm 81,4$ & $148,2 \pm 64,1$ \\
\hline
\end{tabular}

IMC: Índice de Massa Corporal; PAs: Pressão Arterial Sistólica; PAd: Pressão Arterial Diastólica ${ }^{*} \mathrm{p}<0,05$
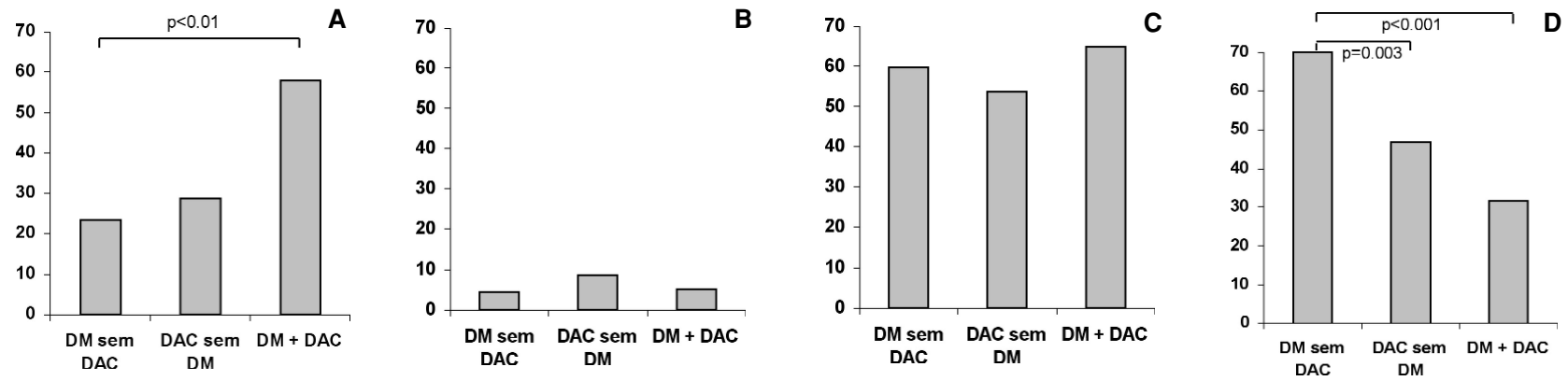

Figura 2. Percentual de pacientes que apresentam os valores adequados do perfil lipídico, conforme recomendação das III Diretrizes Brasileiras de Dislipidemias e Prevenção da Aterosclerose (Triglicérides < $150 \mathrm{mg} / \mathrm{dl}$ (1C), LDL < 100 mg/dl (1A) e $\mathrm{HDL} \geq 45 \mathrm{mg} / \mathrm{dl}$ (1D)) (ref. 10) e do National Cholesterol Education Program Adult Treatment Panel III Guidelines (LDL $<70$ $\mathrm{mg} / \mathrm{dl}(1 \mathrm{~B})$ ) (ref. 11).

este tipo de medicação. Pacientes sem hipolipemiantes $(\mathrm{n}=254)$ apresentavam níveis mais elevados de colesterol total $(213,0 \pm 47,0$ vs. $96,0 \pm 46,0 ; \mathrm{p}<0,001)$, LDL colesterol $(132,0 \pm 41,0$ vs. $116,0 \pm 39,9 ; \mathrm{p}<$ $0,001)$ e HDL colesterol $(53,0 \pm 14,0 \times 46,0$ vs. 12,0 ; $\mathrm{p}<0,001)$, além de menores níveis de triglicérides $(145,0 \pm 87,0$ vs. $160,0 \pm 79,0 ; \mathrm{p}<0,001)$.

$\mathrm{Na}$ tabela 3 são apresentados os valores do perfil lipídico e do IMC antes do início da terapia com estatinas e no último exame apresentado pelo paciente. $\mathrm{O}$ uso de medicamentos esteve associado a uma redução significativa nos valores do colesterol total, LDL colesterol, HDL colesterol e triglicérides. Contudo, as médias do colesterol total, LDL-colesterol e triglicérides encontram-se superiores às metas propostas para a prevenção da aterosclerose em pacientes de alto risco. É interessante notar que não houve variação do IMC neste período. Como apenas um pequeno número de pacientes utilizava exclusivamente fibratos $(n=14)$, optamos por não realizar a análise estatística deste grupo.
Foram também analisados dados referentes à utilização das estatinas. Aproximadamente $72 \%$ da população utilizava a sinvastatina, com uma dose média de 29,8 \pm 12,6 mg/paciente/dia. Em segundo lugar, veio a atorvastatina, correspondendo a $16 \%$ da população (dose média de $18,0 \pm 16,7 \mathrm{mg}$ /paciente) dia). A pravastatina, fluvastatina e lovastatina foram utilizadas apenas por um pequeno percentual de pacientes $(5,0 \%, 3,5 \%$ e $3,5 \%$, respectivamente).

\section{DISCUSSĀO}

Pacientes com DM tipo 2 e/ou DAC apresentam um maior risco de mortalidade decorrente de um evento cardiovascular (IAM ou AVC). Embora seja recomendado que os fatores de risco cardiovasculares, principalmente a dislipidemia, sejam agressivamente tratados nestes pacientes $(11,12)$, isto nem sempre é observado na prática clínica. Através de um levantamento de prontuários, investigamos a abordagem de diversos 
Tabela 3. Perfil lipídico e IMC antes e depois do tratamento hipolipemiante.

\begin{tabular}{lcc}
\hline & $\begin{array}{c}\text { Exames pré-tratamento } \\
\text { com hipolipemiante }\end{array}$ & $\begin{array}{c}\text { Exames durante o uso } \\
\text { de hipolipemiantes }\end{array}$ \\
\hline Colesterol Total $(\mathrm{mg} / \mathrm{dl})$ & $251,0 \pm 40,0$ & $196,0 \pm 46,0^{*}$ \\
$\mathrm{LDL}$ Colesterol $(\mathrm{mg} / \mathrm{dl})$ & $168,0 \pm 36,0$ & $116,0 \pm 39,0^{*}$ \\
$\mathrm{HDL}$ Colesterol $(\mathrm{mg} / \mathrm{dl})$ & $51,0 \pm 46,0$ & $46,0 \pm 12,0^{*}$ \\
Triglicérides $(\mathrm{mg} / \mathrm{dl})$ & $181,0 \pm 120,0$ & $160,0 \pm 79,0^{*}$ \\
$\mathrm{IMC}\left(\mathrm{kg} / \mathrm{m}^{2}\right)$ & $29,2 \pm 4,3$ & $29,3 \pm 4,4$ \\
\hline $\mathrm{IMC}:$ Índice de Massa Corporal & & \\
${ }^{*} \mathrm{p}<0,001$ & &
\end{tabular}

fatores de risco nestes pacientes em centros públicos de referência no Brasil e Venezuela. As principais conclusões foram: 1) pacientes com DM e sem DAC apresentaram valores mais elevados de pressão arterial diastólica, colesterol total, LDL e HDL colesterol do que pacientes com DAC; 2 ) o uso de medicamentos hipolipemiantes, em particular as estatinas, associou-se a uma diminuição dos níveis de colesterol total, LDL colesterol, HDL colesterol e triglicérides; 3) apenas um pequeno percentual dos pacientes, mesmo que em uso de estatina, apresentou os valores do perfil lipídico entre os atualmente recomendados para a prevenção de eventos cardiovasculares $(10,12)$.

A presença de DM tipo 2 é um importante fator de risco para a ocorrência de um evento cardiovascular. Haffner e cols. (13) já demonstraram que o risco de apresentar um IAM é semelhante entre um paciente que já apresentou história prévia de IAM e um paciente com DM tipo 2 sem DAC. Mais ainda, pacientes diabéticos com doença coronária apresentam taxa de mortalidade que pode chegar a $40 \%$ em 5 anos se não tratados adequadamente (11). Desta forma, pressupõe-se que estes pacientes deveriam ser igualmente tratados. Em nossa população, pacientes com DM e sem DAC apresentaram níveis mais elevados de colesterol total, LDL colesterol e pressão arterial diastólica. Estes resultados indicam que existe a necessidade de se melhorar o tratamento dos fatores de risco cardiovasculares nessa população. Interessantemente, os pacientes com DM e sem DAC apresentavam níveis mais elevados de HDL colesterol. Este resultado pode indicar uma menor propensão a doenças cardiovasculares deste grupo, já que o HDL é um possível fator de proteção $(14,15)$. São necessários estudos prospectivos adicionais de modo a confirmar melhor esta hipótese.

Um dos aspectos mais evidentes em nosso estudo foi a falta de uma abordagem adequada aos pacientes diabéticos em relação aos fatores de risco cardiovascular. Embora não tivessem o diagnóstico de DAC, pacientes diabéticos já são considerados como pacientes de alto risco cardiovascular (13) e possuem metas específicas de tratamento. É importante observar que, mesmo quando existe a disponibilidade de estatinas, apenas um pequeno percentual de pacientes atinge os níveis desejados de LDL colesterol $(<100$ $\mathrm{mg} / \mathrm{dl}$ ). Os profissionais que lidam com pacientes diabéticos precisam se conscientizar de que estes pacientes devem ser agressivamente tratados para que a prevenção primária de eventos cardiovasculares seja adequadamente realizada. Embora a utilização de estatinas nestes pacientes já produza benefícios, é necessário que suas doses sejam tituladas de modo a atingir a redução desejada ou que outras drogas sejam associadas, com o objetivo primário de reduzir os níveis de LDL colesterol, mas também atingindo os valores recomendados de triglicérides e HDL colesterol.

Existem cada vez mais evidências sugerindo que a simples presença de intolerância a glicose no jejum (IGJ) já pode ser considerada um fator de risco cardiovascular (ver referências 16 e 17 para revisão). Embora não faça parte dos objetivos deste protocolo, alguns achados importantes foram observados nos pacientes com DAC e sem DM, porém com IGJ. Em comparação aos pacientes com glicemia normal, pacientes com IGJ apresentavam níveis mais reduzidos de LDL colesterol, provavelmente associados a maior utilização de estatinas. Duas hipóteses podem justificar estes achados: (i) pacientes com IGJ já vêm sendo mais agressivamente tratados do que pacientes com glicemia normal, justificando o maior uso de estatinas e os menores níveis de LDL colesterol; (ii) os pacientes com IGJ poderiam apresentar, previamente ao tratamento, níveis mais elevados de LDL colesterol, confirmando seu maior risco cardiovascular. Por possuírem estes níveis mais elevados, estes pacientes receberam estatinas com mais freqüência. Devido, entretanto, à pequena, e muito específica, amostra utilizada, estes achados não puderam ser confirmados.

O impacto da terapia hipolipemiante, em particular as estatinas, no HDL colesterol observado neste estudo não foi semelhante ao descrito na literatura. Diversos estudos clínicos randomizados e controlados 
por placebo (clinical trials) já demonstraram que o uso de estatinas está associado a um aumento nos níveis de HDL colesterol $(18,19)$. Embora estes estudos forneçam evidências controladas da ação das drogas, eles apresentam grandes diferenças com a prática clínica diária dos grandes hospitais. Em nossa população, embora o uso de estatinas esteja associado a uma queda nos níveis de colesterol total, LDL colesterol e triglicérides, existiu também uma queda nos níveis de HDL colesterol em torno de 10\%. A provável ausência de um acompanhamento nutricional regular e de estímulo à prática de atividade física (quase sempre obrigatória nos clinical trials) associado a um possível uso irregular da medicação e a natureza retrospectiva de nosso estudo podem justificar parcialmente este achado. Contudo, apesar das restrições acima, consideramos extremamente benéficos os efeitos das estatinas sobre o LDL colesterol em nossos pacientes. De fato, Dean e cols. (20), numa grande metanálise dos estudos que avaliaram os efeitos das estatinas na prevenção cardiovascular, mostraram que o efeito desses fármacos nos níveis de HDL colesterol é discreto e que o benefício nos estudos clínicos não pode ser atribuído a esse fato, mas sim à redução do LDL colesterol.

Apesar da extrema valorização do controle dos lípides para redução do risco cardiovascular na literatura científica, apenas uma pequena porcentagem dos pacientes atinge os níveis recomendados do LDL e HDL colesterol para a prevenção da DAC (12). Em nosso estudo, aparentemente apenas os pacientes com DM tipo 2 e DAC parecem receber o tratamento de maneira a atingir um LDL colesterol $<100 \mathrm{mg} / \mathrm{dL}$. Se considerarmos hoje a grande prevalência do DM tipo 2 e da DAC isoladamente, é preocupante que, em centros de referência, apenas entre 20 e $30 \%$ dos pacientes tenham um LDL colesterol adequado. Se, por outro lado, forem observadas as novas recomendações do ATP III (LDL $<70 \mathrm{mg} / \mathrm{dl}$ ) (11) para portadores de DAC e DM tipo 2, ou DAC e outros fatores de risco associados, a porcentagem de pacientes que atingem estes níveis é praticamente desprezível (4 a 9\%). Da mesma forma, é importante observar que a grande maioria dos pacientes com DAC (independente da presença de DM) apresenta níveis inadequados de HDL colesterol. Embora do ponto de vista terapêutico o aumento do HDL colesterol não seja ainda considerado tão importante quanto a redução do LDL colesterol, já que apenas o Coronary Drug Project (14) e o estudo VA-HIT (15) mostraram que o aumento do primeiro diminui o risco de eventos cardiovasculares, não há dúvidas de que a combinação de HDL baixo com LDL elevado indica uma maior predis- posição à doença aterosclerótica (6). Dessa forma, esses indivíduos devem ser submetidos a tratamentos preventivos agressivos.

Algumas conclusões importantes podem ser obtidas com este estudo. Primeiro, a necessidade evidente de se reduzir os níveis de LDL nesta população de risco. Talvez o baixo nível sócio econômico dos pacientes avaliados seja um fator que limite a obtenção de um controle adequado do perfil lipídico, apesar de uma parcela importante da população avaliada (mais de $50 \%$ ) neste estudo receber a medicação gratuitamente do Estado. Esse fato sugere ou uma falta de aderência ao tratamento ou um descuido dos médicos em relação ao perfil lipídico, como já previamente demonstrado em nosso meio (21).

Embora possa ser sugerido que um aumento da dose das estatinas poderia ser suficiente para reduzir os níveis de LDL para as metas terapêuticas em nossa casuística, é interessante notar que a dose média da população estudada é relativamente elevada, correspondendo a quase $50 \%$ da dose máxima no caso da sinvastatina. Um fato importante a ser citado é que maior parte do efeito hipolipemiante das estatinas ocorre em doses iniciais, e que quando se dobra a dose do medicamento em média se atinge uma redução adicional do LDL colesterol de apenas 6\% (5). Esse fato fala a favor do uso de estatinas mais potentes ou da associação com outros hipolipemiantes para o tratamento. De fato, a presença de HDL baixo e de triglicérides elevados na grande maioria da população, principalmente nos pacientes em uso de estatina, sugere que, nos pacientes de alto risco, existe uma necessidade crescente de se utilizar outras drogas que interfiram nestes parâmetros, como os fibratos ou a niacina $(22,23)$. Talvez com a maior utilização destas associações, seja possível aumentar o percentual de pacientes com níveis adequados de colesterol total, HDL e LDL colesterol e triglicérides e, desta forma, reduzir ainda mais os eventos cardiovasculares.

Pelo fato de nosso estudo ser de natureza retrospectiva, não aleatória e sem um controle adequado da adesão ao tratamento, nossos resultados necessitam ser avaliados dentro de suas limitações. Entretanto, como o objetivo era o de investigar o efeito dos hipolipemiantes na prática clínica real e num ambiente não controlado, este pareceu ser o desenho de estudo mais adequado. Além disso, todas as informações colhidas foram de variáveis objetivas, de modo a evitar interpretações por parte dos responsáveis pela coleta dos dados.

Apesar de se tratar de um levantamento de prontuários, importantes conclusões podem ser evidenciadas neste estudo. Primeiramente, existe uma clara necessidade de se melhorar a abordagem dos 
pacientes com alto risco para um evento cardiovascular. Embora um percentual importante de pacientes esteja em uso de hipolipemiantes, a maioria possui níveis lipídicos fora do recomendado. É preciso conscientizar os profissionais envolvidos da necessidade de se utilizar inicialmente doses maiores de estatinas, ou dar preferência a estatinas mais potentes (24) ou se associar estatinas a ezetimiba nos casos de LDL colesterol acima das metas (25). Naqueles pacientes com HDL baixo e/ou triglicérides elevados, a associação com niacina ou fibratos pode ser benéfica, desde que se siga o paciente com maior cuidado devido a um infreqüente, contudo possível, maior risco de efeitos colaterais, especialmente os musculares (26).

\section{REFERÊNCIAS}

1. Breslow JL. Cardiovascular disease burden increases, $\mathrm{NIH}$ funding decreases. Nat Med 1997;3:600-1.

2. Braunwald E. Shattuck Lecture - cardiovascular medicine at the turn of the millennium: triumphs, concerns, and opportunities. N Engl J Med 1997:337:1360-9.

3. Fanning EL. Evidence-based disease management: its role in cardiovascular risk reduction. Dis Manag 2004; 7(suppl. 1):S7-10.

4. Kreisberg RA, Oberman A. Lipids and atherosclerosis: lessons learned from randomized controlled trials of lipid lowering and other relevant studies. J Clin Endocrinol Metab 2002;87:423-37.

5. Kreisberg RA, Oberman A. Medical management of hyperlipidemia/dyslipidemia. J Clin Endocrinol Metab 2003:88:2445-61.

6. Chapman MJ, Assmann G, Fruchard J-C, Shepherd J Sirtori C; the European Consensus Panel on HDL-C. Raising high-density lipoprotein cholesterol with reduction of cardiovascular risk: the role of nicotinic acid - a position paper developed by the European Consensus Panel on HDL-C. Curr Med Res Opin 2004;20:1253-68.

7. Lugardon S, Lapeyre-Mestre M, Montastruc JL. Upper gastrointestinal adverse drug reactions and cyclo-oxygenase-2 inhibitors (celecoxib and rofecoxib): a case/non-case study from the French Pharmacovigilance Database. Eur J Clin Pharmacol 2004;60:673-7.

8. Andrews $E$, Dombeck M. The role of scientific evidence of risks and benefits in determining risk management policies for medications. Pharmacoepidemiol Drug Saf 2004; 13:599-608.

9. The Expert Committee on the Diagnosis and Classification of Diabetes Mellitus. Report of the Expert Committee on the Diagnosis and Classification of Diabetes Mellitus. Diabetes Care 1997:20:1183-97.

10. Santos RD, Giannini SD, Fonseca FAH, et al. III Diretrizes brasileiras sobre dislipidemias e diretriz de prevenção da aterosclerose do departamento de aterosclerose da sociedade brasileira de cardiologia. Arq Bras Cardiol 2001:77(supl III): 1-48.
11. Grundy SM, Cleeman JI, Merz CNB, Brewer HB, Clark LT, Hunninghake DB, et al. Implications of recent clinical trials for the National Cholesterol Education Program Adult Treatment Panel III Guidelines. Circulation 2004; 1 10:22739.

12. National Cholesterol Education Program (NCEP) Expert Panel on Detection, Evaluation, and Treatment of High Blood Cholesterol in Adults (Adult Treatment Panel III). Third Report of the National Cholesterol Education Program (NCEP) Expert Panel on Detection, Evaluation, and Treatment of High Blood Cholesterol in Adults (Adult Treatment Panel III) final report. Circulation 2002; 106:3143-421.

13. Haffner SM, Lehto S, Ronnemaa T, Pyorala K, Laakso M. Mortality from coronary heart disease in subjects with type 2 diabetes and in nondiabetic subjects with and without prior myocardial infarction. N Engl J Med 1998:339:229-34.

14. Blankenhorn DH, Nessim SA, Johnson RL, Sanmarco ME, Azen SP, Cashin-Hemphill L. Beneficial effects of combined colestipol-niacin therapy on coronary atherosclerosis and coronary venous bypass grafts. JAMA 1987; $257: 3233-40$

15. Robins SJ, Collins D, Wittes JT, Papademetriou V, Deedwania PC, Schaefer EJ, et al. Veterans Affairs High-Density Lipoprotein Intervention Trial. Relation of gemfibrozil treatment and lipid levels with major coronary events: VA-HIT: a randomized controlled trial. JAMA 2001:285:1585-91.

16. Deedwania PC, Fonseca VA. Diabetes, prediabetes, and cardiovascular risk: shifting the paradigm. Am J Med 2005; 1 18:939-47.

17. Caballero AE. Metabolic and vascular abnormalities in subjects at risk for type 2 diabetes: the early start of a dangerous situation. Arch Med Res 2005;36:241-9.

18. Edwards JE, Moore RA. Statins in hypercholesterolaemia: a dose-specific meta-analysis of lipid changes in randomised, double blind trials. BMC Fam Pract 2003;4(1):18.

19. Miller M, Dobs A, Yuan Z, Battisti WP, Borisute $H$ Palmisano J. Effectiveness of simvastatin therapy in raising HDL-C in patients with type 2 diabetes and low HDLC. Curr Med Res Opin 2004;20:1087-94.

20. Dean BB, Borenstein JE, Henning JM, Knight K, Merz CN. Can change in high-density lipoprotein cholesterol levels reduce cardiovascular risk? Am Heart J 2004; 147:966-76.

21. Santos RD, Sposito AC, dos Santos JE, Fonseca FH, Moriguchi EH, Martinez TL, et al. Programa de Avaliação Nacional do Conhecimento sobre Prevenção da Aterosclerose (PANDORA): Como tem sido feito o tratamento das dislipidemias pelos médicos brasileiros. Ara Bras Cardiol 2000;75:289-95.

22. Ascaso JF, Fernandez-Cruz A, Gonzalez Santos P, Hernandez Mijares A, Mangas Rojas A, Millan J, et al. Significance of high density lipoprotein-cholesterol in cardiovascular risk prevention: recommendations of the $\mathrm{HDL}$ Forum. Am J Cardiovasc Drugs 2004;4:299-314.

23. Meyers CD, Kashyap ML. Pharmacologic elevation of high-density lipoproteins: recent insights on mechanism of action and atherosclerosis protection. Curr Opin Cardiol 2004; 19:366-73. 
24. Jones $\mathrm{PH}$, Davidson $\mathrm{MH}$, Stein $\mathrm{EA}$, et al. Comparison of the efficacy and safety of rosuvastatin versus atorvastatin, simvastatin, and pravastatin across doses (STELLAR* Trial). Am J Cardiol 2003:92:152-60.

25. Bays HE, Ose L, Fraser N, Tribble DL, et al. A multicenter, randomized, double-blind, placebo-controlled, factorial design study to evaluate the lipid-altering efficacy and safety profile of the ezetimibe/simvastatin tablet compared with ezetimibe and simvastatin monotherapy in patients with primary hypercholesterolemia. Clin Ther 2004;26:1758-73.
26. Ratz Bravo AE, Tchambaz L, Krahenbuhl-Melcher A, Hess L, Schlienger RG, Krahenbuhl S. Prevalence of potentially severe drug-drug interactions in ambulatory patients with dyslipidaemia receiving HMG-COA reductase inhibitor therapy. Drug Saf 2005;28:263-75.

\section{Endereço para correspondência:}

Rodrigo O. Moreira

Rua Miguel José Mansur 300, apto. 401

36033-250 Juiz de Fora, MG

Fax: (21) 2224-9562

E-mail: rom_br@yahoo.com 